\title{
Detección de triatominos (Hemiptera: Reduviidae) en ambientes domésticos y extradomésticos. Corrientes, Argentina
}

\author{
Detection of triatomines (Hemiptera: Reduviidae) \\ in domiciliary and extra-domiciliary ecotopes. \\ Corrientes, Argentina
}

Miryam Pieri Damborsky 1

María Esther Bar 1

Elena Beatriz Oscherov 1

\footnotetext{
1 Cátedra de Artrópodos, Facultad de Ciencias Exactas y Naturales $y$ Agrimensura, Universidad Nacional del Nordeste. Av. Libertad 5470 , Corrientes 3400, Argentina. mdambor@exa.unne.edu.ar
}

\begin{abstract}
In order to identify intra-and extra-domiciliary triatomines, a study in different counties of Corrientes Province, Argentina, was conducted from 1985 to 1995. Triatoma infestans (Klug, 1834), Triatoma sordida (Stål, 1859), Panstrongylus geniculatus (Latreille, 1811), and Panstrongylus megistus (Burmeister, 1835) were detected in domestic ecotopes. T. infestans and T. sordida were also found in the peridomicile. Triatoma platensis (Neiva, 1913), Psammolestes coreodes (Bergroth, 1911), T. sordida, and Triatoma rubrovaria (Blanchard, 1843) were collected in sylvatic biotopes. T. infestans was confirmed as the predominant species in the domestic environment and was the only species infected with Trypanosoma cruzi-like parasites. T. sordida was most frequent in extra-domiciliary ecotopes, but could be considered capable of colonizing human dwellings.
\end{abstract}

Key words Triatoma; Disease Vectors; Insects; Insect Vectors

Resumen Esta investigación tiene como objetivo identificar los triatominos que predominan en ambientes domésticos y extradomésticos de diferentes departamentos de la Provincia de Corrientes mediante muestreos llevados a cabo desde 1985 hasta 1995. En los ecótopos domiciliarios se detectaron Triatoma infestans (Klug, 1834), Triatoma sordida (Stål, 1859), Panstrongylus geniculatus (Latreille, 1811) y Panstrongylus megistus (Burmeister, 1835). T. infestans y T. sordida se hallaron también en ambientes peridomésticos. Triatoma platensis (Neiva, 1913), Psammolestes coreodes (Bergroth, 1911), T. sordida y Triatoma rubrovaria (Blanchard, 1843) se colectaron en biótopos silvestres. En los domicilios, se confirmó el predominio de T. infestans. Esta especie fue la única parasitada por tripanosomas cruziformes. Si bien T. sordida fue más frecuente en los ecótopos extradomésticos, se demostró su tendencia a colonizar los ambientes domésticos.

Palabras clave Triatoma; Vectores de Enfermedades; Insectos Vectores 
Los vectores del Trypanosoma cruzi, agente causal de la tripanosomiasis americana, se distribuyen en el continente americano entre las latitudes 42o $\mathrm{N}$ y 460 S (Schofield, 1994). En Argentina y países vecinos, el vector doméstico más importante de la enfermedad de Chagas es Triatoma infestans (WHO, 1997).

La reducción en la densidad de $T$. infestans en los domicilios crea un nicho disponible para otros vectores. En Paraguay y en Brasil, se comprobó la tendencia de especies peridomésticas o silvestres a ocupar estos nichos vacantes (Dias, 1988; Rojas-de-Arias, 1996; Ruas Neto \& Krug, 1995). En Uruguay, Rosa \& Salvatella (1995) advierten sobre la presencia de T. rubrovaria en el domicilio y, en Argentina, Bar et al. (1993) y Wisnivesky-Colli et al.(1993) indican el riesgo potencial que representan $T$. sordida y $T$. guasayana (Wygodzinsky \& Abalos, 1949).

El objetivo del presente trabajo fue detectar la presencia de triatominos en domicilio, peridomicilio y biotopos silvestres en diferentes áreas de la Provincia de Corrientes, Argentina.

\section{Materiales y método}

En el marco del Proyecto: Triatominos de la Provincia de Corrientes, se llevó a cabo un muestreo entomológico desde 1985 hasta 1995, en los departamentos Capital, Mercedes, San Luis del Palmar, San Miguel y Empedrado, situados entre 27 o $27^{\prime} 30^{\prime \prime}$ y 29 o $15^{\prime} 40$ " de Latitud Sur y 57o 30' 32” y $58^{\circ} 52^{\prime} 30^{\prime \prime}$ de Longitud Oeste (Figura 1). El área pertenece a la región biogeográfica Neotropical y comprende sectores de las provincias Paranaense, Chaqueña (Distrito Oriental) y del Espinal.

El clima es subtropical, las lluvias superan los $1.500 \mathrm{~mm}$ anuales y la temperatura media varía entre $19^{\circ}$ y $21^{\circ} \mathrm{C}$, con máximas absolutas de $42^{\circ}$ a $46^{\circ} \mathrm{C}$. El paisaje se caracteriza por la presencia de lomadas arenosas, a las que se asocia una planicie ondulada, en las que domina la sabana con pastizales de Andropogon lateralis y Paspalum notatum, relictos de bosques higrófilos poco densos de Schinopsis balansae, Prosopis affinis, Acacia caven, espartillares de Elyonurus muticus y palmares de Butia yatay (Carnevali, 1994). La fauna está representada por numerosos mamíferos, entre ellos marsupiales (Monodelphis sp., Didelphis azarae y D. albiventris), monos (Cebus sp., Allouatta caraya), y Chrysocyon sp., Dasypus sp., Ctenomys sp., Akodon sp., Lagostomus sp., aves (Crypturellus sp., Nothura sp., Ortalis sp., Egretta L., Furnarius cristata, Myopsitta monacha), reptiles (Caiman sp., Ophis sp., Crotalus sp., Tropidurus sp.) y anfibios (Cabrera \& Willink, 1973).

Las viviendas se seleccionaron mediante un muestreo simple al azar. Los integrantes del equipo de investigación analizaron los domicilios y los peridomicilios, localizados a una distancia de 5 a $15 \mathrm{~m}$ de las viviendas, y los biotopos silvestres situados hasta $1.000 \mathrm{~m}$ de las mismas.

La evaluación entomológica se efectuó por el método activo de captura/hora/hombre. Se utilizó como irritante químico Tetrametrina al $0,2 \%$, para provocar la salida de los redúvidos de sus refugios.

En el laboratorio, los insectos se clasificaron por especie, estado evolutivo y sexo (Carcavallo et al., 1997; Lent \& Wygodzinsky, 1979). Las heces de los triatominos se observaron al microscopio (400x) para confirmar la presencia de tripanosomas.

Con el fin de proporcionar a las autoridades de salud un informe de la situación epidemiológica de la Provincia de Corrientes que pueda ser utilizado en la programación de acciones de control en las viviendas rurales, se calcularon los siguientes indicadores entomológicos para T. infestans (WHO, 1991):

- Indice de Infestación (II) = (№ de casas infestadas/№ de casas examinadas) x 100.

- Indice de Infección Natural (IIN) $=$ (№ de triatominos infectados por T. cruzi/№ de insectos examinados) x 100 .

- Indice de Dispersión (IDD) = (№ de localidades infestadas/№ de localidades examinadas) $\mathrm{x} 100$.

- Indice de Colonización (IC) = (№ de viviendas con ninfas/№ de casas infestadas) x 100 .

- Indice de Densidad (ID) = (№ de triatominos capturados/№ de casas infestadas)

Se compararon los II e IIN obtenidos en las distintas áreas, aplicando el test $\chi^{2}$ a un nivel de significación del $5 \%$.

\section{Resultados}

Se detectaron 4.302 triatominos de las siguientes especies: T. infestans, T. sordida, T. platensis, T. rubrovaria, Panstrongylus megistus, Pa. geniculatus y Psammolestes coreodes.

El total de viviendas analizadas fue de 431, de las cuales 56 estaban ubicadas en áreas urbanas del Departamento Capital y 375 en áreas rurales de los restantes departamentos. La población humana en las áreas muestreadas fue de 2.208 personas. La migración temporaria de grupos familiares por razones laborales a zonas de mayor endemicidad chagásica es fre- 
cuente. La economía de los pobladores se basa principalmente en el cultivo, en pequeñas parcelas, de maíz, tabaco, algodón, hortalizas, destinados al sustento familiar.

La infestación domiciliaria por triatominos fue de $40,1 \%$. El número de viviendas infestadas y los triatominos colectados, discriminados por departamento, se detallan en la Tabla 1.

Se examinaron 214 peridomicilios (depósitos, corrales de aves y de porcinos, pilas de leña, palomares, nidales), de los cuales el 6,5 \% estaban infestados. En los ecótopos peridomésticos, se capturaron 2.409 triatominos (Tabla 2).

El 55,1\% de los biotopos silvestres resultaron infestados por un total de 571 triatominos (Tabla 3). Se revisaron 135 biotopos: nidos de aves, madrigueras, canteras de piedra laja, huecos de árboles. La mayoría de los triatominos se colectaron en nidos de Furnariidae, Psittacidae, Tyrannidae y Emberizidae.

\section{Triatoma infestans}

Esta especie se detectó en todos los departamentos. Su presencia fue predominante en el intradomicilio y, en menor proporción, se localizó también en el peridomicilio.

En la ciudad de Corrientes, Departamento Capital, se hallaron ejemplares de T. infestans en dos viviendas y se detectaron indicadores de infestación (corion y deyecciones) en las paredes de cinco dormitorios. De las 75 viviendas rurales investigadas en Mercedes, sólo en dos se verificó la presencia de T. infestans. Todos los estados evolutivos de esta especie se capturaron en unidades intradomiciliarias de San Luis del Palmar, San Miguel y Empedrado. En San Luis del Palmar y Empedrado se localizó $T$. infestans también en anexos a las viviendas y en corrales de aves.

Los indicadores entomológicos obtenidos para esta especie, en los distintos departamentos, se presentan en la Tabla 4. El II fue mayor en San Miguel, y los IIN, IDD, IC e ID resultaron más elevados en San Luis del Palmar. Los valores de los II e IIN difieren significativamente en los cinco departamentos $\left(\chi^{2}=74,6 ; \mathrm{gl}=4\right.$; $\mathrm{p}=0,0001$ y $\chi^{2}=18,4 ; \mathrm{gl}=4 ; \mathrm{p}=0,001$, respectivamente).

\section{Triatoma sordida}

Se verificó su presencia en ecótopos domésticos, peridomésticos y silvestres, tanto en áreas urbanas como rurales.

En 31 viviendas de Capital se capturaron adultos en número de uno a cuatro por domicilio. La población más numerosa $(\mathrm{n}=1.856)$ se
Figura 1

Ubicación geográfica de los departamentos investigados.

Provincia de Corrientes, Argentina.

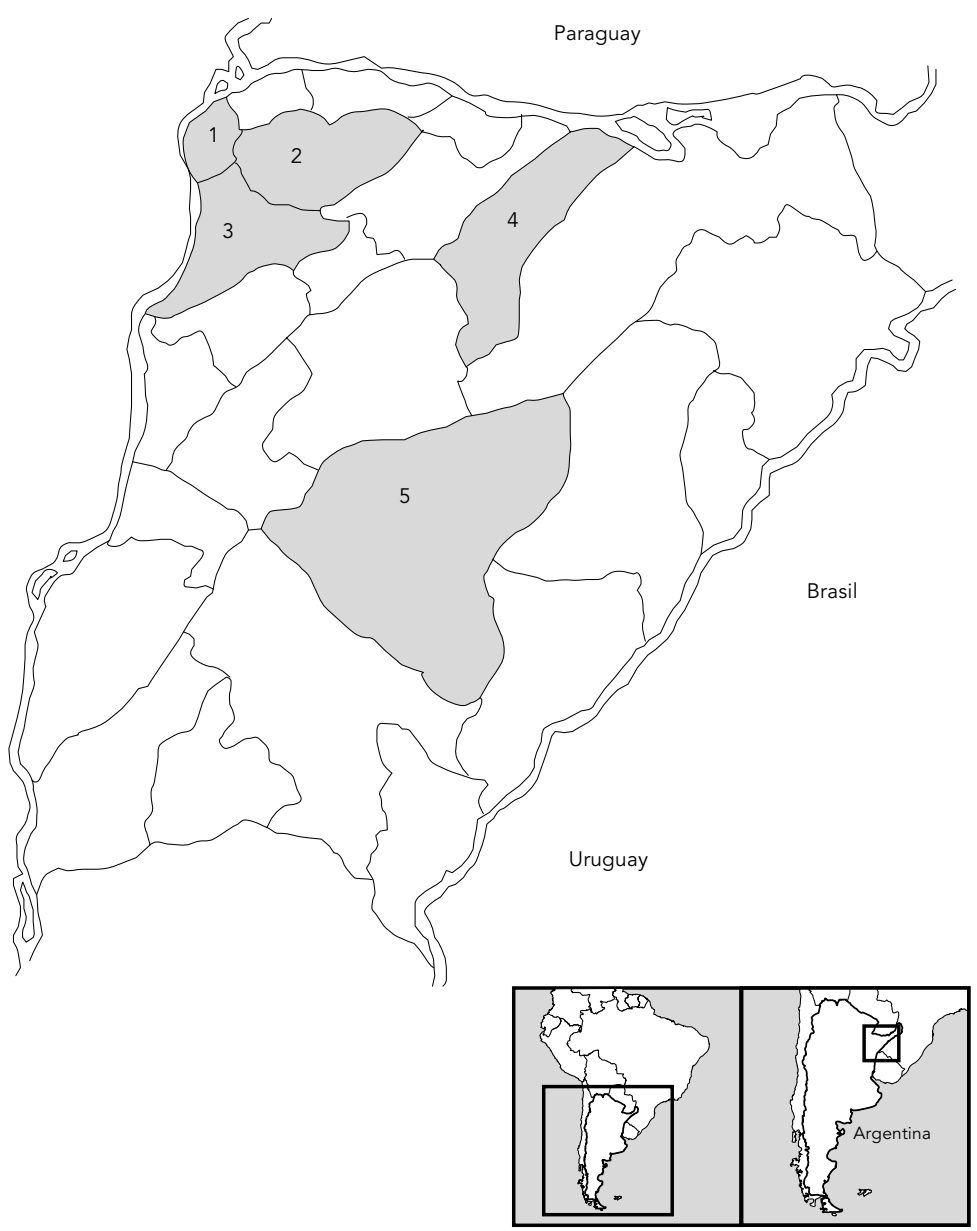

Departamentos: 1 - Capital; 2 - San Luis del Palmar; 3 - Empedrado; 4 - San Miguel; 5 - Mercedes.

capturó en un palomar ubicado en el Parque Mitre de la ciudad de Corrientes. En un domicilio de Empedrado y en dos de San Luis del Palmar se colectaron ejemplares, sin que se verifique colonización en ninguno de ellos.

En las áreas rurales se localizó T. sordida en galpones, pilas de troncos y colonizando corrales de aves. En ecótopos silvestres, tales como nidos de aves y huecos de árboles, se colectaron ninfas de distintos estadios.

Se verificó la convivencia de $T$. sordida con T. infestans en nidales de gallinas y de T. sordi$d a$ con Ps. coreodes y T. platensis en nidos de aves (pájaros). 
Presencia de triatominos en el domicilio en departamentos de la Provincia de Corrientes, Argentina. 1985-1995.

\begin{tabular}{|c|c|c|c|c|c|c|c|c|c|c|}
\hline \multirow[t]{3}{*}{ Departamentos } & \multirow[t]{3}{*}{ VI/VE* } & \multicolumn{8}{|c|}{$\begin{array}{c}\text { Especies colectadas } \\
\text { (no de ejemplares y estados de desarrollo) }\end{array}$} & \multirow[t]{3}{*}{ Total } \\
\hline & & \multicolumn{3}{|c|}{ T. infestans } & \multirow{2}{*}{$\begin{array}{c}\text { Pa. megistus } \\
\text { A }\end{array}$} & \multicolumn{3}{|c|}{ T. sordida } & \multirow{2}{*}{$\begin{array}{c}\text { Pa. geniculatus } \\
\text { A }\end{array}$} & \\
\hline & & $\mathrm{H}$ & $\mathrm{N}$ & $A$ & & $\mathrm{H}$ & $\mathrm{N}$ & $A$ & & \\
\hline Capital & $41 / 56$ & - & - & 2 & - & 13 & 34 & 42 & 3 & 94 \\
\hline Mercedes & $3 / 75$ & - & - & 5 & 1 & - & - & - & - & 6 \\
\hline San Luis del Palmar & $48 / 100$ & 289 & 253 & 119 & - & - & - & 2 & - & 663 \\
\hline San Miguel & $51 / 100$ & 78 & 220 & 57 & - & 35 & 6 & 32 & - & 428 \\
\hline Empedrado & $30 / 100$ & 69 & 31 & 32 & - & - & - & 1 & - & 133 \\
\hline Total & $173 / 431$ & 436 & 504 & 215 & 1 & 48 & 40 & 77 & 3 & 1.324 \\
\hline
\end{tabular}

* = № de viviendas infestadas/№ de viviendas examinadas

$\mathrm{H}=$ Huevos; $\mathrm{N}=$ Ninfas; $\mathrm{A}=$ Adultos

Tabla 2

Triatominos colectados en ecótopos peridomésticos, discriminados por estado evolutivo. Departamentos de la Provincia de Corrientes, Argentina. 1985-1995.

\begin{tabular}{|c|c|c|c|c|c|}
\hline \multirow{2}{*}{$\begin{array}{l}\text { Departamento/ } \\
\text { Especie }\end{array}$} & \multirow[t]{2}{*}{ Tipo de ecótopo } & \multicolumn{3}{|c|}{ Estado evolutivo } & \multirow[t]{2}{*}{ Total } \\
\hline & & Huevos & Ninfas & Adultos & \\
\hline \multicolumn{6}{|l|}{ Capital } \\
\hline T. sordida & Pila de troncos & - & 84 & 12 & 96 \\
\hline T. sordida & Palomar & 6 & 1.827 & 23 & 1.856 \\
\hline T. sordida & Depósitos & & 33 & 3 & 36 \\
\hline \multicolumn{6}{|l|}{ Mercedes } \\
\hline T. sordida & Pila de leña & - & - & 3 & 3 \\
\hline \multicolumn{6}{|c|}{ San Luis del Palmar } \\
\hline T. sordida & Corral de aves & 3 & 305 & 2 & 310 \\
\hline T. sordida & Nidal de gallinas & - & 24 & - & 24 \\
\hline T. infestans & Nidal de gallinas & & 2 & 1 & 3 \\
\hline T. infestans & Depósitos & 46 & 7 & 4 & 57 \\
\hline \multicolumn{6}{|l|}{ Empedrado } \\
\hline T. sordida & Corral de aves & - & 21 & 2 & 23 \\
\hline \multirow[t]{2}{*}{ T. infestans } & Corral de aves & - & - & 1 & 1 \\
\hline & & & & & 2.409 \\
\hline
\end{tabular}

\section{Triatoma rubrovaria}

Se colectaron ninfas en una cantera correspondiente a afloramientos de areniscas, en el Departamento Mercedes. Los triatominos estaban refugiados en lajas acumuladas y preparadas para su transporte a centros urbanos, donde son utilizadas en la construcción de viviendas. En primavera, se constató la invasión de adultos a las viviendas de los jornaleros que trabajaban en la cantera y que habitan en las inmediaciones.

\section{Triatoma platensis y Psammolestes coreodes}

Ambas especies se hallaron sólo en nidos de aves de Capital, Mercedes, San Luis del Palmar y San Miguel. Se capturaron ejemplares de todos los estados evolutivos de T. platensis en el $13,2 \%$ de los nidos y, en $39,5 \%$ de los mismos, se comprobó la presencia de Ps. coreodes.

\section{Panstrongylus geniculatus y Panstrongylus megistus}

Ejemplares adultos de Pa. geniculatus se capturaron en tres domicilios de la ciudad de Corrientes y un ejemplar adulto de $\mathrm{Pa}$. megistus fue capturado en una vivienda rural de Mercedes. Estos triatominos llegaron por medio del vuelo, atraídos por la luz artificial.

\section{Discusión}

El tipo de construcción de las viviendas rurales fue semejante, sin embargo no se verificaron índices de infestación similares. Las paredes eran de "estanteo" (troncos y/o "cañas": Gadua sp., unidos con una mezcla de tierra, agua y pasto, etc.) y los techos de "paja" (Panicum prionitis), mientras que los domicilios urbanos tenían paredes de mampostería y techos de zinc.

En Capital y Mercedes, el hallazgo de T. infestans fue esporádico, no se comprobó colonización ni infección en los triatominos; asimismo, los II, IDD e ID en estos departamentos 
fueron los más bajos. En Mercedes, este hecho podría atribuírse al uso de insecticidas de alto poder residual (Bar \& Oscherov, 1985/ 1986).

Los indicadores entomológicos de T. infestans obtenidos en San Luis del Palmar señalan una alta infección por tripanosomas cruziformes de estos triatominos. Asimismo, la amplia dispersión de la especie en las localidades investigadas y el elevado número de ninfas en los domicilios indicarían que los habitantes de este departamento son los que están expuestos a mayor riesgo de adquirir la infección chagásica.

T. rubrovaria es rupestre, y sus ecótopos naturales son las áreas de afloramiento granítico. En la Provincia de Corrientes, Mercedes es el único departamento que presenta estas características geológicas, lo que explicaría su ausencia en los restantes departamentos.

Las piedras lajas de un sector de la cantera donde fue hallada T. rubrovaria representan un foco de dispersión pasiva. Esta especie es mencionada como vector secundario, sustituto de T. infestans en Uruguay, debido a su capacidad para colonizar el intradomicilio, donde alcanza altas densidades poblacionales (Salvatella et al., 1994).

T. platensis ha sido encontrada infectada por Tr. cruzi en condiciones naturales (Giraldez et al., 1997; Lent \& Wygodzinsky, 1979). En el área estudiada, se la halló asociada a nidos de aves. La presencia de D. albiventris en algunos nidos positivos a T. platensis indica que esta especie cuenta entre sus hospederos potenciales a este reservorio del Tr. cruzi (Schweigmann, 1994). Se hace notar que son necesarios estudios más específicos que posibiliten conocer si T. platensis interviene en el ciclo silvestre del Tr. cruzi en la zona.

En el área estudiada, T. platensis, T. rubrovaria y Ps. coreodes se colectaron únicamente en ecótopos silvestres. Tanto estos triatominos como Pa. megistus y Pa. geniculatus carecen, por el momento, de importancia epidemiológica, ya que no se comprobó colonización del domicilio, ni infección por Tr. cruzi en los ejemplares capturados.

Una colonia de 73 ejemplares de T. sordida, constituída principalmente por huevos y adultos, fue detectada en una vivienda rural de San Miguel (Bar et al., 1996a). Resulta interesante expresar que la vivienda había sido fumigada en dos oportunidades en la semana previa al muestreo.

T. sordida fue identificada como vector de Tr. cruzi en el Chaco paraguayo (Rojas de Arias et al., 1993) y, en Argentina, Bar et al. (1996b) informan sobre el hallazgo de ejemplares infectados por Tr. cruzi en ecótopos silvestres.
Tabla 3

Triatominos colectados en ecótopos silvestres, discriminados por estado evolutivo. Departamentos de la Provincia de Corrientes, Argentina, 1985-1995.

\begin{tabular}{|c|c|c|c|c|}
\hline \multirow{2}{*}{$\begin{array}{l}\text { Departamentos/ } \\
\text { Especies colectadas }\end{array}$} & \multicolumn{3}{|c|}{ Estado evolutivo } & \multirow[t]{2}{*}{ Total } \\
\hline & Huevos & Ninfas & Adultos & \\
\hline \multicolumn{5}{|l|}{ Capital } \\
\hline Ps. coreodes & - & 29 & 18 & 47 \\
\hline T. platensis & - & 5 & 1 & 6 \\
\hline \multicolumn{5}{|l|}{ Mercedes } \\
\hline Ps. coreodes & - & 3 & 51 & 54 \\
\hline T. platensis & - & 20 & 10 & 30 \\
\hline T. rubrovaria & - & 81 & - & 81 \\
\hline \multicolumn{5}{|l|}{ San Luis del Palmar } \\
\hline Ps. coreodes & 101 & 38 & 100 & 239 \\
\hline T. platensis & - & 40 & 9 & 49 \\
\hline \multicolumn{5}{|l|}{ San Miguel } \\
\hline Ps. coreodes & - & 4 & - & 4 \\
\hline T. platensis & - & 2 & - & 2 \\
\hline T. sordida & - & 39 & - & 39 \\
\hline \multicolumn{5}{|l|}{ Empedrado } \\
\hline \multirow[t]{2}{*}{ T. sordida } & - & 20 & - & 20 \\
\hline & & & & 571 \\
\hline
\end{tabular}

Tabla 4

Indicadores entomológicos de Triatoma infestans obtenidos en distintos departamentos de la Provincia de Corrientes, Argentina, 1985 - 1995.

\begin{tabular}{lccccc}
\hline Departamentos & \multicolumn{5}{c}{ Indices entomológicos } \\
& II & IIN & IDD & IC & ID \\
\hline Capital & 12,5 & - & 28,6 & - & 0,04 \\
Mercedes & 2,7 & - & 25 & - & 0,07 \\
San Luis del Palmar & 46 & 38,6 & 87,5 & 54,3 & 13,8 \\
San Miguel & 50 & 23,1 & 66,6 & 38 & 6,9 \\
Empedrado & 29 & 23,1 & 54,5 & 31 & 4,4 \\
\hline
\end{tabular}

II = Indice de Infestación; IIN = Indice de Infección Natural;

IDD = Indice de Dispersión; IC = Indice de Colonización; ID = Indice de Densidad

La presencia de T. sordida en todos los ambientes demuestra una amplia valencia ecológica en relación con las restantes especies secundarias halladas. El elevado número capturado en un ecótopo urbano de la ciudad de Corrientes, así como en el interior de las viviendas, plantea la posibilidad de que T. sordida ocupe el nicho vacío dejado por T. infestans en el do- 
micilio. Por otro lado, el hallazgo de ejemplares infectados permite considerar a esta especie como el vector secundario de mayor importancia epidemiológica en la Provincia de Corrientes.

T. infestans conserva su carácter de colonizadora del intradomicilio, con porcentajes importantes de infestación e infección natural, por lo que se confirma su rol como vector principal de la enfermedad de Chagas.

Cabe resaltar que, en los departamentos San Luis del Palmar, San Miguel y Empedrado, se

\section{Agradecimentos}

Trabajo financiado por la Secretaría General de Ciencia y Técnica, Universidad Nacional del Nordeste.

\section{Referencias}

BAR, M. E. \& OSCHEROV, E. B., 1985/1986. Triatomismo domiciliario de la provincia de Corrientes, $\mathrm{Ar}$ gentina. II. Areas rurales del Departamento de Mercedes. Facena, 6:57-66.

BAR, M. E.; OSCHEROV, E. B.; ALVAREZ, B. M.; DAMBORSKY, M. P.; MIZDRAJI, G.; PORCEL, E. \& AVALOS, G., 1996a. Infestación doméstica por Triatoma infestans y prevalencia de seropositivos al Trypanosoma cruzi en un área rural del Nordeste Argentino. Revista da Sociedade Brasileira de Medicina Tropical, 29:549-555.

BAR, M. E.; OSCHEROV, E. B.; AVALOS, G.; DAMBORSKY, M. P. \& ALVAREZ, B. M., 1996b. Infección natural de Triatoma sordida por Trypanosoma cruzi en ecótopos silvestres de la provincia de Corrientes, Argentina. In: 5ta. Jornada de Biología, Resúmenes, p. 32. Asunción: Instituto de Investigaciones en Ciencias de la Salud, Universidad Nacional de Asunción.

BAR, M. E.; OSCHEROV, E. B. \& DAMBORSKY, M. P., 1993. Presencia de Triatoma sordida Stal, 1859 en ecotopos urbanos de la ciudad de Corrientes, Argentina. Revista de Saúde Pública, 27:117-122.

BAR, M. E., OSCHEROV, E. B.; DAMBORSKY, M. P. VARELA, M. E.; MIZDRAJI, G. \& PORCEL, E., 1992. Triatomismo del Departamento San Luis del Palmar de la Provincia de Corrientes, Argentina. $\mathrm{Me}$ dicina (Buenos Aires), 52:193-201.

BAR, M. E.; DAMBORSKY, M. P.; OSCHEROV, E. B.; ALVAREZ, B. M.; MIZDRAJI, G. \& AVALOS, G., 1997. Infestación domiciliaria por triatominos y seroprevalencia humana en el Departamento Empe- constató una seroprevalencia humana al $\mathrm{Tr}$. cruzi que osciló entre 22,3\% y 32,3\% (Bar et al., 1992, 1996a, 1997). Esta situación y también la carencia de un programa de control vectorial sistemático y las bajas condiciones socioeconómicas y culturales de los pobladores de las áreas estudiadas reflejan el riesgo epidemiológico al que están expuestos. Estos factores actuarían como responsables del mantenimiento de la endemia chagásica en la provincia. drado, Corrientes, Argentina. Cadernos de Saúde Pública, 13:37-45.

CABRERA, A. L. \& WILLINK, A., 1973. Biogeografía de América Latina. Serie de Biología, Monografía № 13. Washington, DC: Programa Regional de Desarrollo Científico y Tecnológico, Departamento de Asuntos Científicos, Secretaría General, Organización de Estados Americanos.

CARCAVALLO, R. U.; GALINDEZ-GIRON I.; JURBERG, J.; GALVAO C. \& LENT, H., 1997. Pictorial keys for tribes, genera \& species of the subfamily triatominae. In: Atlas of Chagas' Disease Vectors in the Americas (R. U. Carcavallo, I. Galindez-Giron, J. Jurberg \& H. Lent, ed.), v. 1, pp. 107-244, Rio de Janeiro: Editora Fiocruz.

CARNEVALI, R., 1994. Fitogeografía de la Provincia de Corrientes. Corrientes: Gobierno de la Provincia de Corrientes/Instituto Nacional de Tecnología Agropecuaria/Editorial Del Autor.

DIAS, J. C. P., 1988. Controle de vetores da doença de Chagas no Brasil e riscos de reinvasão domiciliar por vetores secundários. Memórias do Instituto Oswaldo Cruz, 83:387-391.

GIRALDEZ, E.; ROVERANO, R. \& REMONTE, M., 1997. Presencia de Triatoma platensis, Neiva 1913, infectados en las inmediaciones de la vivienda humana. Medicina (Buenos Aires), 55:84.

LENT, H. \& WYGODZINSKY, P., 1979. Revision of the triatominae (Hemiptera: Reduviidae), and their significance as vectors of Chagas' disease. Bulletin of the American Museum of Natural History, 163:123-520. 
ROJAS-DE-ARIAS, A. 1996. Chagas' disease in Paraguay. Washington, DC: Pan-American Health Organization, World Health Organization.

ROJAS-DE-ARIAS, A.; GUILLEN, I.; INCHAUSTI, A.; SAMUDIO, M. \& SCHMEDA-HIRSCHMANN, G., 1993. Prevalence of Chagas' disease in Ayoreo communities of the Paraguayan Chaco. Tropical Medicine Parasitology, 44:285-288.

ROSA, R. \& SALVATELLA, R., 1995. Diagnóstico de situación epidemiológica actual de la enfermedad de Chagas en Uruguay. Boletín de la Sociedad de Zoología de Uruguay, 9:6-11.

RUAS NETO, A. L. \& KRUG, L., 1995. Epidemiologia da doença de Chagas no Rio Grande do Sul: A distribução vetorial. Boletín de la Sociedad de Zoología de Uruguay, 9:20-32.

SALVATELLA, R.; CALEGARI, L.; PUIME, A.; BASMADJIAN, Y.; ROSA, R.; GUERRERO, J.; MARTINEZ, M.; MENDARO, G.; BRIANO, D.; MONTERO, C. \& WISNIVESKY-COLLI, C., 1994. Perfil alimentario de Triatoma rubrovaria (Blanchard, 1843) (Hemiptera, Triatominae) en ámbitos peridomiciliarios de una localidad rural de Uruguay. Revista do Instituto de Medicina Tropical de São Paulo, 36:311-320.
SCHOFIELD, C. J., 1994. Triatominae: Biología y Control. West Sussex: Eurocommunica Publications.

SCHWEIGMANN, N. J., 1994. Aspectos Ecológicos de una Población Santiagueña de la Comadreja Overa (Didelphis albiventris) en Relación con la Transmisión de Trypanosoma cruzi. Tesis de Doctorado, Buenos Aires: Facultad de Ciencias Exactas, Universidad de Buenos Aires.

WHO (World Health Organization), 1991. Control of Chagas disease. WHO Technical Report Series 811. Geneva: WHO.

WHO (World Health Organization), 1997. Tropical Disease Research: Prospects for Elimination. Geneva: WHO.

WISNIVESKY-COLLI, C.; GÜRTLER, R. E.; SOLARZ, N. D.; SCHWEIGMANN, N. J.; PIETROKOVSKY, S. M.; ALBERTI, A. \& FLO, J., 1993. Dispersive flight and house invasion by Triatoma guasayana and Triatoma sordida in Argentina. Memórias do Instituto Oswaldo Cruz, 88:27-32. 